\title{
A placebo-controlled investigation of synaesthesia-like experiences under LSD
}

Devin B. Terhune

Department of Experimental Psychology, University of Oxford, Oxford, UK

Department of Psychology, Goldsmiths, University of London, London, UK

David P. Luke

Department of Psychology \& Counselling, University of Greenwich, London, UK

Mendel Kaelen

Centre for Neuropsychopharmacology, Division of Brain Sciences, Faculty of Medicine, Imperial College London, London, UK

Mark Bolstridge

Centre for Neuropsychopharmacology, Division of Brain Sciences, Faculty of Medicine, Imperial College London, London, UK

Amanda Feilding

The Beckley Foundation, Beckley Park, Oxford, UK

David Nutt

Centre for Neuropsychopharmacology, Division of Brain Sciences, Faculty of Medicine, Imperial College London, London, UK

Robin Carhart-Harris

Centre for Neuropsychopharmacology, Division of Brain Sciences, Faculty of Medicine, Imperial College

London, London, UK

Jamie Ward

Department of Psychology, University of Sussex, Sussex, UK

\section{Correspondence:}

Devin B. Terhune

Department of Psychology, Goldsmiths, University of London, London, UK

Email: d.terhune@gold.ac.uk

Robin Carhart-Harris

Centre for Neuropsychopharmacology, Division of Brain Sciences, Faculty of Medicine, Imperial College London, London, UK

Email: r.carhart-harris@imperial.ac.uk

Jamie Ward

Department of Psychology, University of Sussex, Sussex, UK

Email: jamiew@sussex.ac.uk 


\section{Abstract}

The induction of synaesthesia in non-synaesthetes has the potential to illuminate the mechanisms that contribute to the development of this condition and the shaping of its phenomenology. Previous research suggests that lysergic acid diethylamide (LSD) reliably induces synaesthesia-like experiences in nonsynaesthetes. However, these studies suffer from a number of methodological limitations including lack of a placebo control and the absence of rigorous measures used to test established criteria for genuine synaesthesia. Here we report a pilot study that aimed to circumvent these limitations. We conducted a within-groups placebo-controlled investigation of the impact of LSD on colour experiences in response to standardized graphemes and sounds and the consistency and specificity of grapheme- and sound-colour associations. Participants reported more spontaneous synaesthesia-like experiences under LSD, relative to placebo, but did not differ across conditions in colour experiences in response to inducers, consistency of stimulus-colour associations, or in inducer specificity. Further analyses suggest that individual differences in a number of these effects were associated with the propensity to experience states of absorption in one's daily life. Although preliminary, the present study suggests that LSD-induced synaesthesia-like experiences do not exhibit consistency or inducer-specificity and thus do not meet two widely established criteria for genuine synaesthesia.

Keywords: colour; consciousness; consistency; hallucinogens; psychedelics; serotonin; 


\section{Introduction}

Synaesthesia is a neurological condition characterized by atypical binding in which a stimulus (inducer) will reliably and involuntarily elicit a secondary conscious experience (concurrent) (Ward, 2013). In congenital synaesthetes, for example, a particular number or auditory tone may automatically elicit a specific colour experience. Accumulating evidence from familial studies suggests that synaesthesia is hereditary (Barnett et al., 2008; Baron-Cohen, Burt, Smith-Laittan, Harrison, \& Bolton, 1996) and there is preliminary research pointing to possible genetic polymorphisms underlying this condition (Asher et al., 2009; Tomson et al., 2011). In contrast, the available evidence suggests that the particular inducerconcurrent pairings that one experiences may be driven by environmental constraints (Witthoft \& Winawer, 2013). Delineating the mechanisms underlying the development of synaesthesia has the potential to inform our broader understanding of numerous facets of cognition and perception including automaticity, binding, memory, and sensory processing (Cohen Kadosh \& Henik, 2007; Ward, 2013).

In recent years, there has been increased attention to the possibility of inducing synaesthesia or synaesthesia-like perceptual states in non-synaesthetes (Brogaard, 2013; Deroy \& Spence, 2013; Luke \& Terhune, 2013). One of the more striking instances of this includes the apparent induction through chemical agents of experiences that closely resemble synaesthesia. Indeed, there is reliable evidence that a number of different drugs, particularly tryptamines, which largely function as serotonin agonists (Nichols, 2004), including lysergic acid diethylamide (LSD), psilocybin, and ayahausca, produce experiences that closely resemble synaesthesia, including the experience of colour in response to sounds (Hartman \& Hollister, 1963; Kelly, 1934; Masters \& Houston, 1966; Simpson \& McKellar, 1955). However, a systematic review of these studies revealed that they suffered from numerous methodological limitations including the absence of placebo controls and the failure to use established behavioural measures of different features of synaesthesia including automaticity, inducer specificity, and consistency of inducerconcurrent associations (Luke \& Terhune, 2013). For these reasons, controversy persists as to whether these drug-induced experiences qualify as genuine synaesthesia (Deroy \& Spence, 2013; Luke \& Terhune, 2013; Sinke et al., 2012). 
Although complete consensus has not yet fully emerged regarding the adjudication criteria by which a phenomenon is identified as synaesthesia (e.g., Simner, 2012), the majority of researchers agree that an experience qualifies as synaesthesia if the inducer-concurrent mappings are (a) automatic; (b) consistent; (c) specific; and (d) accessible to consciousness (Ward, 2013). The aforementioned review (Luke \& Terhune, 2013) found that research investigating the chemical induction of synaesthesia-like experiences had not formally tested whether any of the first three criteria were met, thus rendering it unclear how similar these phenomena are to genuine synaesthesia.

The present study sought to replicate previous reports of LSD-induced synaesthesia-like experiences and determine whether they met the adjudication criteria of consistency and specificity. LSD was selected for this study because a survey of drug-users previously found that this drug was associated with the highest frequency of induced synaesthesia (Luke, Terhune, \& Friday, 2012). Toward this end, as part of a larger placebo-controlled validation study of the feasibility of studying LSD in a neuroimaging environment (Carhart-Harris et al., 2015), participants rated the vividness of colour experiences and selected specific colours in response to a set of graphemes and sounds in LSD and placebo conditions. Participants also independently completed measures of absorption and visual imagery (Blajenkova, Kozhevnikov, \& Motes, 2006; Jamieson, 2005; Ott, 2007; Tellegen \& Atkinson, 1974), which may be related to the experience of drug-induced synaesthesia-like experiences (Studerus, Gamma, Kometer, \& Vollenweider, 2012). We expected that LSD would produce synaesthesia-like experiences, particularly for sound stimuli, to a greater degree than placebo. We also sought to explore whether these experiences differed in consistency and inducer-specificity across conditions.

\section{Methods}

This study used a within-groups design involving the administration of LSD and a placebo on separate days. Here we report crucial features of the present study. The full methodological details of the study have been described in detail elsewhere (Carhart-Harris et al., 2015). 


\subsection{Participants.}

A convenience sample of 10 participants (one female; $M_{\mathrm{Age}}=34.2 \pm 7.4$, range $=26-47$ ) consented to participate in this study. Participants were over the age of 21 , did not have personal or immediate family history of a psychiatric illness, did not have a history of drug or alcohol dependence, or a medical condition that might render them unsuitable for participation in the study, based on self-reported information disclosed to a team psychiatrist. All participants had prior experience with a classic psychedelic drug, such as LSD, mescaline, or ayahuasca; this criterion was included to minimize the possibility of adverse responses to the drug. All participants were considered mentally and physically healthy in an examination by the psychiatrist. The study was approved by the NRES committee London, West London and was conducted in accordance with the revised Declaration of Helsinki (2000), the International Committee on Harmonisation Good Clinical Practice guidelines, and the NHS Research Governance Framework. Imperial College London sponsored the research and a Home Office license was obtained for research with a schedule one drug. Participants were informed that the study sought to determine an appropriate dose of LSD for a neuroimaging study and would involve completing a battery of psychological tasks.

\subsection{Materials}

\subsubsection{Pre-experimental self-report measures.}

Participants completed a synaesthesia screening and two psychological measures prior to the experiment.

\section{Synaesthesia screening.}

Participants completed a questionnaire that defined synaesthesia and included examples of specific forms of synaesthesia and were asked whether they had any forms of synaesthesia. They were also asked whether they had experienced synaesthesia under the influence of any drugs, which drugs, and the frequency (rarely, some of the time, most of the time, every time), and the type of synaesthesia. 


\section{Psychological absorption.}

In a separate session, participants completed the modified version (MODTAS; Jamieson, 2005) of the Tellegen Absorption Scale (Tellegen \& Atkinson, 1974). This 34-item scale taps a range of experiences pertaining to one's propensity for having states of absorption characterized by intense attentional, affective, and perceptual involvement in activities. A representative item includes: "When I listen to music, I can get so caught up in it that I don't notice anything else." Items are scored on a 5-point likert scale with anchors of "never" and "very often". The Cronbach's $\alpha$ for this sample was .96, indicating strong internal consistency.

\section{Visual imagery.}

Participants also completed the Object-Spatial Imagery Questionnaire (OSIQ; Blajenkova et al., 2006), a 30-item scale with a 5-point response format ("totally disagree" to "totally agree"). The OSIQ includes two 15-item subscales measuring object ("My images are very colourful and bright.") and spatial ("I was very good in 3-D geometry as a student.”) visual imagery, both of which had good internal consistency ( $\alpha \mathrm{s}=.86$ and .88 , respectively).

\subsubsection{Experimental measures.}

Participants completed the following tasks and measures in each drug condition.

\section{Grapheme-colour associations.}

This task assessed the vividness of any colour experienced in response to graphemes and the consistency of grapheme-colour associations. Participants received the following instructions: "After looking at each symbol, choose a colour. If you experienced a colour whilst looking at the symbol then choose the colour nearest to that. If you didn't experience a colour then guess which colour might go best with that symbol." Each trial consisted of a $1 \mathrm{~s}$ fixation cross (in black against a gray background), followed by a $2 \mathrm{~s}$ presentation of a grapheme (in black). Participants subsequently selected a colour from a $30 \times 12$ colour 
palette and then judged their colour experience "What was your COLOUR experience when you saw the symbol?" using the Perceptual Awareness Scale (PAS; Sandberg, Timmermans, Overgaard, \& Cleeremans, 2010), a four-point likert-type measure (1: "No experience"; 2: "Brief glimpse"; 3: "Almost clear image"; and 4: "Absolutely clear image"). Participants had unlimited time for the colour selection and experience judgment. Blocks consisted of 25 unique symbols: the digits 0-9 and the 15 most frequent letters in the English language (a, c, d, e, h, I, 1, m, n, o, r, s, t, u, w; Lewand, 2000) presented in random order. After a 2 s inter-block interval, participants completed a second block of trials with the same symbols in a different random order.

\section{Sound-colour associations.}

This task was formatted in the same way as the grapheme-colour association task, but included 24 unique sounds including eight vowel sounds (Moos, Smith, Miller, \& Simmons, 2014); six pure tones (100, 200, $400,800,1600,3200 \mathrm{~Hz})$; two further pure tone sounds $(400 \mathrm{~Hz})$ that were louder and quieter; six complex sounds made up of a noise element $(100-3200 \mathrm{~Hz}$ bandpassed noise) and a tonal element (made up of sinusoids from $100 \mathrm{~Hz}$ to $3200 \mathrm{~Hz}$ in a geometric series) with the relative loudness of the noise varying; and two rapidly shifting sounds (one of which was the noise of a magnetic resonance imaging (MRI) T2 scan sequence). These sounds were previously used in studies of crossmodal sound-colour correspondences (Hamilton-Fletcher, 2015). Auditory stimuli were presented to participants using sandstorm SBS2112 sound system with the speakers placed in front of the participants

\section{Post-task questionnaire.}

To explore spontaneous synaesthesia-like experiences beyond colour, participants answered eight questions, administered in random order, after each task using a visual analogue scale with anchors at "strongly disagree" (0) and "strongly agree" (1) (see Table 1). They were subsequently asked if the graphemes/sounds triggered visual experiences that resembled a shown image (using the same scale). They were shown eight images consisting of six patterns linked by Klüver (Klüver, 1966), amongst others, 
to psychedelic experiences (lattices, parallel lines, dots and flecks, zigzags, nested curves, and spirals) and two images that were included as controls (line drawings of faces and a rural landscape).

\subsection{Procedure}

Study sessions were conducted in the Wellcome Trust Clinical Research Facility (WTCRF) at the Hammersmith Hospital site, London. Participation involved two sessions separated by 5-7 days with placebo administered on the first visit (to avoid potential carry-over effects from LSD into the placebo condition); participants were unaware of the order and blind to the condition. Each testing session started between 10 and 11a.m.; participants were first briefed about the procedure and completed a urine test for drugs of abuse and a breathalyzer test for alcohol use. A medical doctor inserted and secured a cannula into a vein in the antecubital fossa and blood pressure was measured prior to dosing. Participants were encouraged to close their eyes and relax in a reclined position prior to the administration of a 10-ml solution of saline alone (placebo) or containing LSD (40-80 $\mu \mathrm{g}$; one participant received 40, two 50, six 70 , and one $80 \mu \mathrm{g}$ ) intravenously infused over a $3 \mathrm{~min}$ period. Heart rate was measured throughout each testing session and recorded at 1-5 min intervals for the first 45 min post-infusion (monitoring phase); an experimenter also recorded self-ratings of the subjective intensity of the drug effects on an 11-point scale (0: "no effects" to 10: "extremely intense effects"). Participants subsequently completed the battery of psychological tasks, which included the present tasks and others (Carhart-Harris et al., 2015; Kaelen et al., 2015); heart rate and subjective ratings were recorded every 30-45 min for the remainder of the session. Stimulus presentation and response recording for the tasks was implemented with Psychopy v. 1.80.01 (Peirce, 2007, 2008) on a HP 9470m notebook with a 14in. monitor. Participants started the tasks $170 \pm 9 \mathrm{~min}$ after drug administration; at that point, participants report drug intensity effects of $0 \pm 0$ and $6.1 \pm 0.8$ in the placebo and LSD conditions, respectively. Participants were assessed by a psychiatrist for suitability of discharge after the subjective effects of LSD had sufficiently subsided (approximately 5-6 h post-infusion). 


\subsection{Analyses}

\section{Inducer specificity}

To assess inducer specificity (i.e., the extent to which the same inducers produced colours on different occasions), we computed a measure of how reliably inducers elicited reports of colour experiences in the grapheme- and sound- colour tasks. Specifically, we calculated the absolute difference between colour experience (PAS) ratings for each stimulus pair (i.e., in block 1 and block 2) in each condition and task. In order to focus on subjective colour experiences, only stimulus pairs in which one member of each pair received a PAS rating of 2 ("brief glimpse") or above were included; lower values reflect greater correspondence between colour experience ratings across the two presentations (i.e., greater inducer specificity). The proportion of stimulus ratings that were excluded from these analyses was greater $(M \pm S E)$ in the grapheme task (placebo: $.54 \pm .10$; LSD: $.57 \pm .12$ ) than in the sound task (placebo: $.39 \pm .12$; LSD: $.32 \pm .12), F(1,9)=5.38, p=.045, \eta_{\mathrm{p}}{ }^{2}=.37$, although proportions did not differ across conditions, $F(1,9)=0.08, p=.78, \eta_{\mathrm{p}}{ }^{2}=.01$, and there was no Condition $\times$ Task interaction $F(1,9)=1.40, p=.27, \eta_{\mathrm{p}}{ }^{2}=.13$.

\section{Stimulus-colour consistency}

Stimulus-colour consistency (i.e., the reliability with which graphemes and sounds were associated with the same colours on different trials) was indexed using an established measure of inducer-concurrent consistency (Eagleman, Kagan, Nelson, Sagaram, \& Sarma, 2007). This measure is computed by averaging the geometric distance in RGB colour space for RGB values for each pair of stimuli presented across the two blocks in the sound and colour tasks (see also Rothen, Seth, Witzel, \& Ward, 2013). Lower values reflect less colour distance across stimulus presentations and thus greater stimulus-colour consistency.

\section{Within-participant regression analyses}

These analyses sought to determine and whether, and to what extent, the experience of colour in response to graphemes and sounds was associated with greater grapheme- and sound- colour consistency. We 
performed within-participant regression analyses in which consistency scores for each stimulus pair were regressed on the mean PAS scores (across the two presentations of a stimulus). This yielded an individual beta coefficient for each task and condition for each participant; negative coefficients indicate that greater colour awareness was associated with lower stimulus-colour consistency scores (reflecting greater consistency).

\section{Statistical Analyses}

All analyses were performed in MATLAB (2014a; The MathWorks Inc., Natick, MA, USA) or SPSS (v. 20; IBM). Data were normally distributed (Kolmogorov-Smirnov test) in the inducer-specificity and inducer-concurrent consistency analyses. One or more variables included in the analyses of colour experiences, in the within-participant regression analyses, and in the analyses self-reported spontaneous experiences violated the assumption of distribution normality, primarily because of negatively skewed data. In all cases except self-reports of smell experiences, these violations were corrected through log transformations. Colour experience data (PAS scores) were pooled across blocks. Data were analyzed with 2 (Condition: placebo v. LSD) $\times 2$ (Task: grapheme v. sound) repeated measures ANOVAs. Data were further analyzed using $2 \times 2$ ANCOVAs with the same factors and including self-report measures (see above) as continuous covariates in order to follow-up on previous research suggesting that such variables may moderate drug-induced synaesthesia (Studerus et al., 2012).

\section{Results}

\subsection{Prior experience of drug-induced synaesthesia-like experiences}

Three of the 10 participants reported prior experience of drug-induced synaesthesia-like experiences. One reported sound-colour synaesthesia on psilocybin and ayahuasca; another reported sound-colour synaesthesia on LSD, and grapheme-colour synaesthesia on psilocybin; the third reported sound-colour synaesthesia on 2,5-dimethoxy-4-bromophenethylamine (2C-B), LSD, and ketamine. All three reported that these experiences occurred "some of the time". One participant self-reported congenital grapheme- 
colour synaesthesia, but their data were in the range of the remaining participants in both placebo and LSD conditions and so were not treated differently.

\subsection{Stimulus-specific colour experiences under LSD}

The first analysis contrasted colour experiences in the different drug conditions and tasks using the PAS in a 2 (Condition: placebo v. LSD) $\times 2$ (Task: grapheme v. sound) repeated measures ANOVA. Colour reports were numerically higher in the LSD condition, but there were neither main effects of Condition, $F(1,9)=1.52, p=.25, \eta_{\mathrm{p}}{ }^{2}=.14$, or Task, $F(1,9)=2.16, p=.18, \eta_{\mathrm{p}}{ }^{2}=.19$, nor an interaction $F(1,9)=0.19, p=.67$, $\eta_{\mathrm{p}}{ }^{2}=.02$ (Fig. 1a). The inclusion of MODTAS and OSIQ subscale scores as covariates in an ANCOVA did not change this pattern and none of the measures was a significant covariate. These results suggest that participants did not report more colour experiences in response to graphemes and sounds in the LSD condition. 

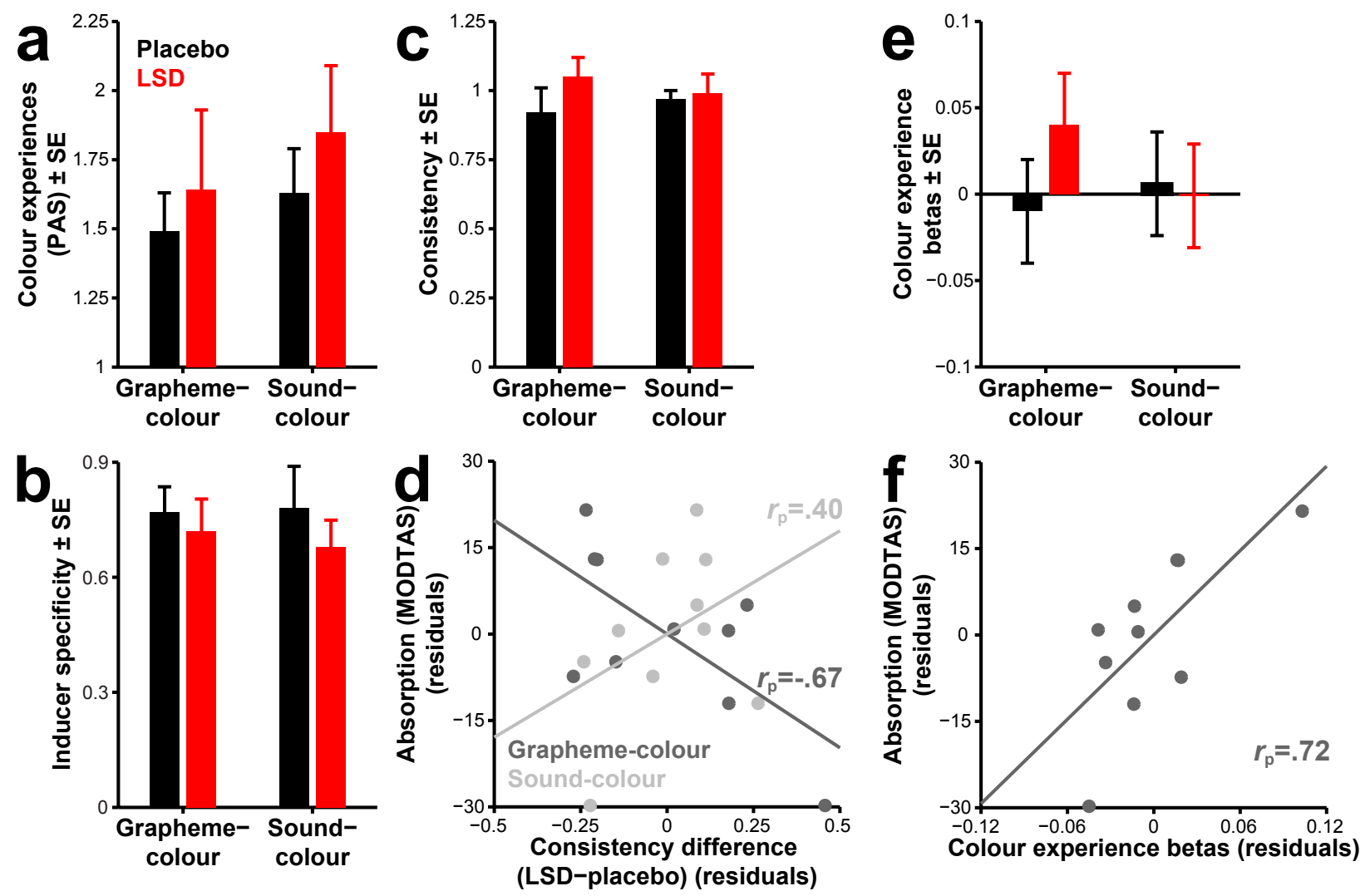

Figure 1. Grapheme-colour and sound-colour association parameters as a function of drug. (a) Colour experiences (PAS); (b) Inducer specificity (average absolute PAS difference scores between stimulus presentations [lower values reflect greater specificity]); (c) Consistency of stimulus-colour associations (lower values reflect greater consistency); (d) Absorption (MODTAS) differentially correlated with LSDplacebo differences in grapheme-colour and sound-colour consistency (residuals controlling for OSIQ object and spatial subscales); (e) Within-participant colour experience beta coefficients (in the prediction of stimulus-colour consistency) (negative values indicate that colour experience was associated with lower consistency scores [greater consistency]); (f) Absorption (MODTAS) positively correlated with colour experience beta coefficients collapsed across conditions (controlling for OSIQ object and spatial subscales) indicating that higher absorption is associated with a tendency for colour experience and consistency scores to be positively related [a negative association between colour experience and consistency].

\subsection{Inducer specificity}

A core feature of congenital synaesthesia is that specific inducers will elicit particular concurrents. Here we sought to examine whether specific inducers consistently produced colour experiences. In the index of inducer specificity we developed, lower values reflect greater correspondence between colour experiences across the different presentations of a stimulus in a particular condition (greater specificity). Insofar as this analysis focused on inducers that elicited subjective colour experience (according to the PAS), we 
excluded stimuli in which participants did not report any colour experiences on both presentations of the stimulus; accordingly, three participants were excluded from the analysis because they reliably had no colour experiences in at least one condition. As can be seen in Fig. 1b, inducer specificity scores were numerically lower in the LSD condition, but there were no main effects of Condition, $F(1,6)=0.70, p=.44$, $\eta_{\mathrm{p}}{ }^{2}=.10$, or Task, $F(1,6)=0.01, p=.92, \eta_{\mathrm{p}}{ }^{2}<.01$, nor an interaction, $F(1,6)=0.48, p=.52, \eta_{\mathrm{p}}{ }^{2}=.07$. The inclusion of the self-report measures as covariates in an ANCOVA did not change this pattern and none of the measures was a significant covariate. This suggests that inducer-specificity did not change under LSD.

\subsection{Grapheme-colour and sound-colour consistency}

If LSD was inducing genuine synaesthesia, one might expect that grapheme-colour and sound-colour associations would be more consistent under LSD in comparison with placebo, as is observed in congenital synaesthetes relative to non-synaesthete controls (Eagleman et al., 2007; Rothen et al., 2013). Using an established measure of consistency (Eagleman et al., 2007), for which lower scores reflect greater consistency, participants displayed numerically higher scores in the LSD condition, but there were neither main effects of Condition, $F(1,9)=2.77, p=.13, \eta_{\mathrm{p}}{ }^{2}=.24$, or Task, $F(1,9)=.01, p=.93, \eta_{\mathrm{p}}{ }^{2}<.01$, nor an interaction, $F(1,9)=0.75, p=.41, \eta_{\mathrm{p}}{ }^{2}=.08$ (Fig. 1c). When the three self-report measures were included as covariates, there was a Drug $\times$ Task $\times$ MODTAS interaction, $F(1,6)=6.93, p=.039, \eta_{\mathrm{p}}{ }^{2}=.54$. This indicated that the tendency for LSD to increase grapheme-colour consistency scores (reflecting a decrease in consistency) relative to placebo, was more strongly correlated with a propensity for states of absorption, $r_{\mathrm{p}}=-.67, p=.069$, than the corresponding change in sound-colour consistency scores, $r_{\mathrm{p}}=.40$, $p=.33$ (Fig. 1d).

A limitation of the foregoing analysis is that participants did not report synaesthetic experiences for all stimuli and changes in consistency scores could be blunted by the inclusion of inducer-concurrent pairings with and without concurrent colour experiences. Thus, we analyzed beta coefficients from within-participant regression analyses in which consistency scores were regressed on PAS scores. There 
were no main effects of Drug, $F(1,9)=0.68, p=.43, \eta_{\mathrm{p}}{ }^{2}=.07$, or Task, $F(1,9)=0.26, p=.62, \eta_{\mathrm{p}}{ }^{2}=.03$, nor a Drug $\times$ Task interaction on beta coefficients, $F(1,9)=1.38, p=.27, \eta_{\mathrm{p}}{ }^{2}=.13$ (Fig. 1e). Individual beta coefficients ranged from -.16 to .19 and across participants did not differ from 0 in any of the conditions, $t \mathrm{~s}(9)<1.6, p \mathrm{~s}>.16$, suggesting that the experience of colours in response to graphemes and sounds was unrelated to stimulus-colour mapping consistency. When this analysis was repeated including self-report measures as covariates in an ANCOVA, there was a main effect of MODTAS scores, $F(1,6)=6.33$, $p=.046, \eta_{\mathrm{p}}{ }^{2}=.51$ (all other effects remained non-significant), reflecting a positive correlation between beta coefficients (collapsed across conditions) and MODTAS scores, $r_{\mathrm{p}}=.72, p=.046$ (Fig. 1f). This indicates that higher absorption was associated with a tendency for colour awareness to be associated with higher consistency scores (lower stimulus-colour consistency). Cumulatively, these results indicate that synaesthesia-like experiences are not more consistent under LSD than placebo and that stimulus-colour consistency is unrelated to the conscious experience of colours in response to inducers. They further suggest that proneness to absorption states may moderate the impact of different facets of stimulus-colour consistency.

\subsection{Stimulus-specific spontaneous experiences}

Our final analyses concerned eight types of spontaneous synaesthesia-like experiences during the grapheme and sound tasks (see Table 1). There were main effects of Drug on visual movements, $F(1,9)=5.24, p=.048, \eta_{\mathrm{p}}{ }^{2}=.37$, experience of touch, $F(1,9)=6.99, p=.027, \eta_{\mathrm{p}}{ }^{2}=.44$, and Klüver forms, $F(1,9)=8.72, p=.016, \eta_{\mathrm{p}}{ }^{2}=.49$, with each being reported more frequently under LSD. In contrast, there were no Drug effects on visual patterns, $F(1,9)=3.27, p=.10, \eta_{\mathrm{p}}{ }^{2}=.27$, projector-like visual experiences, $F(1,9)=2.31, p=.16, \eta_{\mathrm{p}}{ }^{2}=.20$, or control visual forms (faces and landscapes), $F(1,9)=1.25, p=.29, \eta_{\mathrm{p}}{ }^{2}=.12$. Independent of drug condition, sounds were more likely to elicit novel visual patterns, $F(1,9)=13.65$, $p=.005, \eta_{\mathrm{p}}{ }^{2}=.60$, visual movements, $F(1,9)=7.43, p=.023, \eta_{\mathrm{p}}{ }^{2}=.45$, and associator-like visual images, $F(1,9)=23.20, p=.001, \eta_{\mathrm{p}}{ }^{2}=.72$. All other effects and interactions were non-significant, $F \mathbf{s}<4.8, p \mathrm{~s}>.05$. When the self-report measures (MODTAS and OSIQ) were included as covariates, participants were 
suggestively more likely to report no visual experiences in the placebo condition, $F(1,6)=4.93, p=.068$,

$\eta_{\mathrm{p}}{ }^{2}=.45$, and to report projector-like visual experiences in the LSD condition, $F(1,6)=4.42, p=.080$,

$\eta_{\mathrm{p}}{ }^{2}=.42$. Taken together, these results point to a consistent trend for LSD to elicit stronger spontaneous synaesthesia-like experiences and further indicate that sounds are more likely than graphemes to elicit spontaneous visual experiences.

Table 1.

Descriptive statistics $[M$ and $(S D)]$ for spontaneous experiences as a function of Drug and Task.

\begin{tabular}{|c|c|c|c|c|}
\hline & \multicolumn{2}{|c|}{ Placebo } & \multicolumn{2}{|c|}{ LSD } \\
\hline & Grapheme & Sound & Grapheme & Sound \\
\hline The sounds/symbols triggered visual patterns & $.08(.10)$ & $.26(.24)$ & $.25(.33)$ & $.46(.39)$ \\
\hline The sounds/symbols triggered visual movement & $.03(.05)$ & $.31(.29)$ & $.31(.39)$ & $.46(.40)$ \\
\hline I didn't really have any visual experiences in & $.82(.20)$ & $.68(.34)$ & $.70(.34)$ & $.52(.39)$ \\
\hline \multicolumn{5}{|l|}{ response to the sounds/symbols } \\
\hline The sounds/symbols triggered visual & $.14(.16)$ & $.42(.33)$ & $.27(.38)$ & $.43(.41)$ \\
\hline \multicolumn{5}{|l|}{ experiences in my mind's eyes } \\
\hline The sounds/symbols triggered visual & $.08(.12)$ & $.18(.27)$ & $.18(.32)$ & $.26(.36)$ \\
\hline \multicolumn{5}{|l|}{ experiences that were projected externally } \\
\hline The sounds/symbols triggered touch & $.05(.08)$ & $.10(.19)$ & $.19(.32)$ & $.29(.39)$ \\
\hline The sounds/symbols triggered smells & $.03(.04)$ & $.08(.13)$ & $.07(.17)$ & $.09(.24)$ \\
\hline The sounds/symbols triggered tastes & $.04(.06)$ & $.09(.11)$ & $.07(.16)$ & $.09(.23)$ \\
\hline Klüver forms (e.g., spirals) & $.09(.16)$ & $.27(.24)$ & $.23(.26)$ & $.38(.35)$ \\
\hline Control forms (e.g., faces) & $.08(.13)$ & $.11(.14)$ & $.17(.33)$ & $.12(.21)$ \\
\hline
\end{tabular}

Note. Each item was scored on a visual analogue scale with higher values reflecting stronger endorsement of the respective experience. 


\section{Discussion}

This study sought to expand upon previous reports of LSD-induced synaesthesia-like experiences (Hartman \& Hollister, 1963; Luke \& Terhune, 2013; Masters \& Houston, 1966; Simpson \& McKellar, 1955) by determining whether these experiences meet established criteria for synaesthesia in a withingroups placebo-controlled design. Somewhat surprisingly, but consistent with the extant literature, LSD produced only weak spontaneous synaesthesia-like experiences relative to placebo. Neither consistency of stimulus-colour associations nor inducer specificity differed across drug conditions, suggesting that LSDinduced synaesthesia-like experiences do not meet two established criteria for this condition (Ward, 2013). These results demonstrate that the induction of synaesthesia-like experiences under LSD can be studied in a placebo-controlled design with established measures and hint at directions for further research.

Previous research has documented how LSD and other tryptamines produce spontaneous perceptual states that closely resemble synaesthesia (for a review, see Luke \& Terhune, 2013). In line with these studies, we observed a consistent tendency for participants to report spontaneous synaesthesia-like experiences, but not control percepts (e.g., faces), more frequently in the LSD condition in post-task questionnaires. In contrast, participants did not differ in the extent to which they experienced colours in response to the inducers in the different experimental conditions. In addition, they did not exhibit greater inducer-concurrent consistency or inducer specificity - two widely accepted criteria for synaesthesia - in the LSD condition, relative to the placebo condition. A number of possibilities may explain these discrepancies. First, it is possible that the dosages of LSD were not sufficiently high to produce synaesthesia although this seems unlikely given the dosages used and the drug intensity ratings and spontaneous experiences reported by participants. A second possibility is that participants believed that they had been administered LSD in the first session and then had expectancy-related changes in perceptual states or reported such changes due to demand characteristics, thus blunting any differences between the placebo and LSD conditions. However, none of the participants reported believing they had taken LSD in the placebo condition and self-reported drug intensity ratings in this condition were 
uniformly at 0 at the time of task completion so this again seems highly unlikely. Third, it is plausible that drug-induced synaesthesia-like experiences are superficially similar to, but qualitatively distinct from, congenital synaesthesia and thus should not be expected to meet standard criteria for the latter. The present results are consistent with this interpretation and the related claim that the mechanisms and characteristics of drug-induced synaesthesia-like experiences are different from those of congenital synaesthesia (Deroy \& Spence, 2013; Hubbard \& Ramachandran, 2003; Sinke et al., 2012). This interpretation is perhaps further bolstered by the fact that participants were able to detect which condition they were in (see below) and yet still did not display response patterns similar to congenital synaesthetes.

Another plausible explanation for the discrepant results is that our sample size did not offer sufficient statistical power to observe the induction of synaesthesia under LSD. Indeed, the effects of induced colour experiences and inducer specificity were in the direction predicted if LSD were producing genuine experiences and may have achieved statistical significance in a study with greater power. In contrast, consistency of stimulus-colour associations under LSD was not numerically greater than under placebo, although it is plausible that LSD impairs selective attention or attenuates distractor suppression (Carter et al., 2005; Wapner \& Krus, 1960), which may interfere with response patterns on this task. Insofar as the same participants reported vivid visual experiences in response to music (Kaelen et al., 2015), it could be argued also that richer stimuli are required to induce synaesthesia under LSD than the simple stimuli used here, which are different from the stimuli that typically evoke synaesthesia under the influence of psychedelic drugs (Luke \& Terhune, 2013). However, this may still represent a challenge to the hypothesis that these experiences qualify as synaesthesia because complex stimuli are not required for the induction of synaesthesia in congenital synaesthetes (Ward, 2013). Complicating matters further is that we observed heterogeneity in response to LSD with some participants exhibiting pronounced effects and others displaying only minimal responses. We provide some preliminary evidence that these effects are moderated by proneness to states of absorption, in line with previous research (Studerus et al., 2012). Further research in larger samples using counter-balanced designs, more variable doses, and stimuli of varying complexity will be necessary to better understand these effects. 
A final explanation for our results may be that previous reports of psychedelic drug-induced synaesthesia were related to spontaneous occurrences of this phenomenon rather than its induction via stimuli in a controlled experimental paradigm. We have previously observed negligible effects of psychedelics on perceptual and physiological responses in other stimulus-response paradigms (Muthukumaraswamy et al., 2013), leading us to infer that the predominant (and indeed quintessential) effect of psychedelics is on spontaneous (i.e. on-going) processes, rather than induced responses. This is very much in keeping with the present results where participants reported spontaneous synaesthesia-like experiences. This situation presents a special challenge because stimulus-response paradigms benefit from high experimental control, which is why this approach was adopted here, but also because synaesthesia is typically regarded as a reliable stimulus-specific phenomenon. Unlike congenital synaesthesia, where the inducer plays an essential role, in psychedelic-induced synaesthesia-like experiences, transient fluctuations in perceptual states may be more critical. Certain scenarios (such as the present one) may demand that such experimental control be ceded in order to maintain the conditions in which the predicted phenomenon is intended to occur. Alternative techniques such as experiential-sampling (e.g., Kane et al., 2007) could potentially be used to "capture" drug-induced synaesthesia-like experiences as they arise spontaneously. This approach could also be combined with simultaneous neuroimaging, with the aim of identifying the neural correlates of these phenomena.

Our inducer-concurrent consistency results, in particular, have some potentially important implications. Inducer-concurrent consistency is a widely used, and highly robust, diagnostic marker of congenital synaesthesia (Eagleman et al., 2007; Rothen et al., 2013). In the present study, grapheme- and sound- colour associations did not differ in consistency across LSD and placebo conditions, with associations being actually numerically less consistent in the former condition, a result that is strongly at odds with the hypothesis that LSD is producing consistent synaesthesia-like experiences. We further examined whether experiencing colour in response to inducers was associated with greater stimuluscolour consistency, but found no evidence for this, irrespective of drug condition or task. A notable result was that the propensity for experiencing states of absorption (Jamieson, 2005; Ott, 2007; Tellegen \& 
Atkinson, 1974) appeared to moderate individual differences in a number of facets of stimulus-colour consistency, including the extent to which colour experiences predict stimulus-colour consistency. Although they should be interpreted with caution, these correlations are potentially consistent with previous research showing that absorption predicts the occurrence of LSD-induced synaesthesia-like experiences (Studerus et al., 2012) and evidence indicating that synaesthetes score higher on psychometric measures of absorption (Chun \& Hupe, 2015; Glicksohn, Steinbach, \& ElimalachMalmilyan, 1999; Rader \& Tellegen, 1987) and the related trait of openness to experience (Banissy et al., 2013). However, the present results imply that individuals exhibiting high absorption who experience LSD-induced colours will exhibit reduced stimulus-colour consistency. Moreover, a potential confound that has yet to be addressed to our knowledge is that associations between absorption and synaesthesia or synaesthesia-proneness could be driven by the inclusion of items pertaining to crossmodal correspondences and synaesthesia-like experiences in the self-report measure of absorption, the MODTAS (e.g., Terhune, 2009). Further research on the induction of synaesthesia with pharmacological agents and other methods should follow up on such relations to better determine how absorption may facilitate synaesthesia-like experiences or covary with different facets of this condition.

Cumulatively, the present results suggest that LSD-induced synaesthesia-like experiences do not meet two established criteria for synaesthesia: inducer-specificity and stimulus-colour consistency. Despite the need for caution given the likely under-powered nature of the statistical analyses, the current findings arguably challenge the possibility that LSD is inducing genuine synaesthesia (at least according to standard definitions of this condition) and thus the inclusion of such experiences in taxonomies of synaesthesia (Grossenbacher \& Lovelace, 2001). Aside from a single critique (Simner, 2012), the criterion of inducer-concurrent consistency is perhaps the most well-agreed upon criterion of this condition. Nevertheless, we have previously argued that consistency might be too stringent of a criterion for transient forms, or early stages, of synaesthesia (Luke \& Terhune, 2013). For instance, it is probable that consistency arises from the consolidation of inducer-concurrent pairings over time and thus will not be present at early stages. This hypothesis is consistent with research showing that congenital child 
synaesthetes display lower consistency at earlier developmental stages (Simner \& Bain, 2013; Simner, Harrold, Creed, Monro, \& Foulkes, 2009). The same may apply to inducer-specificity. These factors complicate tests of the "genuineness" of ostensible synaesthesias, particularly those that are transient, and arguably render the absence of consistency and inducer-specificity effects inconclusive. One way of addressing this may be to couple cognitive training in stimulus-colour associations with the administration of LSD, although an early attempt in this direction with mescaline was unsuccessful (Kelly, 1934).

In addition to the small sample size, an important limitation of this study is the ineffectiveness of the blind. In particular, all participants were able to identify which condition they were in (placebo v. LSD), thereby weakening the efficacy of the placebo-controlled design. This limitation is difficult to circumvent as the effects of LSD on conscious awareness are pronounced. Further research may benefit from contrasting LSD with other psychoactive substances that will produce alterations in conscious states but which are not expected to produce synaesthesia-like experiences, such as opiates (Luke et al., 2012). Nevertheless, this limitation arguably strengthens the interpretability of the null results. Specifically, insofar as participants were able to determine the drug condition, the demand characteristics in the LSD condition were probably much higher. Accordingly, the fact that no differences in consistency and inducer-specificity were observed under such circumstances suggests that LSD-induced synaesthesias do not produce such effects, as they were not even able to benefit from participants' expectancies and the experimental demands.

In summary, in a within-groups placebo-controlled study, we observed that LSD did not substantially alter the tendency to experience colour concurrents in response to sounds and graphemes and that stimulus-colour experiences under the influence of LSD did not meet accepted criteria for synaesthesia. Given the sample size of this study, these negative results should not be considered conclusive. Nevertheless, this study demonstrates the possibility of investigating the induction of synaesthesia with pharmacological agents in a controlled setting using rigorous measures. Although preliminary, our results suggest that LSD-induced synaesthesia-like experiences are qualitatively different from congenital 
synaesthesia although they raise potentially important questions regarding the experimental study of such phenomena.

\section{Acknowledgments}

This research received financial and intellectual support from the Beckley Foundation and was conducted as part of a wider Beckley-Imperial research programme. This paper presents independent research carried out at the NIHR/Wellcome Trust Imperial Clinical Research Facility. The views expressed are those of the author(s) and not necessarily those of the NHS, the NIHR or the Department of Health. DBT was supported by a Marie Skłodowska-Curie Intra-European Fellowship within the 7th European Community Framework Programme.

\section{References}

Asher, J. E., Lamb, J. A., Brocklebank, D., Cazier, J. B., Maestrini, E., Addis, L., . . Monaco, A. P. (2009). A whole-genome scan and fine-mapping linkage study of auditory-visual synesthesia reveals evidence of linkage to chromosomes 2q24, 5q33, 6p12, and 12p12. American Journal of Human Genetics, 84(2), 279-285. doi: 10.1016/j.ajhg.2009.01.012

Banissy, M. J., Holle, H., Cassell, J., Annett, L., Tsakanikos, E., Walsh, V., . . W Ward, J. (2013). Personality traits in people with synaesthesia: Do synaesthetes have an atypical personality profile? Personality and Individual Differences, 54(7), 828-831.

Barnett, K. J., Finucane, C., Asher, J. E., Bargary, G., Corvin, A. P., Newell, F. N., \& Mitchell, K. J. (2008). Familial patterns and the origins of individual differences in synaesthesia. Cognition, 106(2), 871-893. doi: 10.1016/j.cognition.2007.05.003

Baron-Cohen, S., Burt, L., Smith-Laittan, F., Harrison, J., \& Bolton, P. (1996). Synaesthesia: Prevalence and familiality. Perception, 25(9), 1073-1079.

Blajenkova, O., Kozhevnikov, M., \& Motes, M. A. (2006). Object-spatial imagery: New self-report imagery questionnaire. Applied Cognitive Psychology, 20(2), 239-263. doi: 10.1002/acp.1182

Brogaard, B. (2013). Serotonergic hyperactivity as a potential factor in developmental, acquired and druginduced synesthesia. Front Hum Neurosci, 7, 657. doi: 10.3389/fnhum.2013.00657 
Carhart-Harris, R. L., Kaelen, M., Whalley, M. G., Bolstridge, M., Feilding, A., \& Nutt, D. J. (2015). LSD enhances suggestibility in healthy volunteers. Psychopharmacology, 232(4), 785-794. doi: 10.1007/s00213-014-3714-z

Carter, O. L., Burr, D. C., Pettigrew, J. D., Wallis, G. M., Hasler, F., \& Vollenweider, F. X. (2005). Using psilocybin to investigate the relationship between attention, working memory, and the serotonin 1A and 2A receptors. Journal of Cognitive Neuroscience, 17(10), 1497-1508. doi: $10.1162 / 089892905774597191$

Chun, C. A., \& Hupe, J. M. (2015). Are synesthetes exceptional beyond their synesthetic associations? A systematic comparison of creativity, personality, cognition, and mental imagery in synesthetes and controls. British Journal of Psychology. doi: 10.1111/bjop.12146

Cohen Kadosh, R., \& Henik, A. (2007). Can synaesthesia research inform cognitive science? Trends Cogn Sci, 11(4), 177-184. doi: 10.1016/j.tics.2007.01.003

Deroy, O., \& Spence, C. (2013). Training, hypnosis, and drugs: Artificial synaesthesia, or artificial paradises? Frontiers in psychology, 4, 660. doi: 10.3389/fpsyg.2013.00660

Eagleman, D. M., Kagan, A. D., Nelson, S. S., Sagaram, D., \& Sarma, A. K. (2007). A standardized test battery for the study of synesthesia. Journal of Neuroscience Methods, 159(1), 139-145. doi: 10.1016/j.jneumeth.2006.07.012

Glicksohn, J., Steinbach, I., \& Elimalach-Malmilyan, S. (1999). Cognitive dedifferentiation in eidetics and synaesthesia: Hunting for the ghost once more. Perception, 28(1), 109-120.

Grossenbacher, P. G., \& Lovelace, C. T. (2001). Mechanisms of synesthesia: Cognitive and physiological constraints. Trends Cogn Sci, 5(1), 36-41.

Hamilton-Fletcher, G. I. D. (2015). How touch and hearing influence visual processing in sensory substitution, synaesthesia and cross-modal correspondences. (PhD), University of Sussex.

Hartman, A. M., \& Hollister, L. E. (1963). Effect of mescaline, lysergic acid diethylamide and psilocybin on color perception. Psychopharmacologia, 4, 441-451. 
Hubbard, E. M., \& Ramachandran, V. S. (2003). Refining the experimental lever - A reply to Shanon and Pribram. Journal of Consciousness Studies, 10(3), 77-84.

Jamieson, G. A. (2005). The modified tellegen absorption scale: A clearer window on the structure and meaning of absorption. Australian Journal of Clinical and Experimental Hypnosis, 33, 119-139.

Kaelen, M., Barrett, F. S., Roseman, L., Lorenz, R., Family, N., Bolstridge, M., . . Carhart-Harris, R. L. (2015). LSD enhances the emotional response to music. Psychopharmacology (Berlin), 232, $3607-3614$.

Kane, M. J., Brown, L. H., McVay, J. C., Silvia, P. J., Myin-Germeys, I., \& Kwapil, T. R. (2007). For whom the mind wanders, and when: An experience-sampling study of working memory and executive control in daily life. Psychol Sci, 18(7), 614-621. doi: 10.1111/j.14679280.2007.01948.x

Kelly, E. L. (1934). An experimental attempt to produce artificial chromaesthesia by the technique of conditioned response. Journal of Experimental Psychology, 17, 315-341.

Klüver, H. (1966). Mescal and mechanisms of hallucinations. Chicago, IL: University of Chicago Press.

Lewand, R. E. (2000). Cryptological mathematics. Mathematical Association of America: Washington, DC.

Luke, D. P., \& Terhune, D. B. (2013). The induction of synaesthesia with chemical agents: A systematic review. Frontiers in psychology, 4, 753. doi: 10.3389/fpsyg.2013.00753

Luke, D. P., Terhune, D. B., \& Friday, R. (2012). Psychedelic synaesthesia: Evidence for a serotonergic role in synaesthesia. Seeing \& Perceiving, 25, 74.

Masters, R. E. L., \& Houston, J. (1966). The varieties of psychedelic experience. London, UK: Turnstone.

Moos, A., Smith, R., Miller, S. R., \& Simmons, D. R. (2014). Cross-modal associations in synaesthesia: Vowel colours in the ear of the beholder. Iperception, 5(2), 132-142. doi: 10.1068/i0626

Muthukumaraswamy, S. D., Carhart-Harris, R. L., Moran, R. J., Brookes, M. J., Williams, T. M., Errtizoe, D., . . Nutt, D. J. (2013). Broadband cortical desynchronization underlies the human 
psychedelic state. Journal of Neuroscience, 33(38), 15171-15183. doi:

10.1523/JNEUROSCI.2063-13.2013

Nichols, D. E. (2004). Hallucinogens. Pharmacology and Therapeutics, 101(2), 131-181. doi: 10.1016/j.pharmthera.2003.11.002

Ott, U. (2007). States of absorption: In search of neurobiological foundations. In G. A. Jamieson (Ed.), Hypnosis and conscious states: The cognitive neuroscience perspective (pp. 257-270). Oxford, UK: Oxford University Press.

Peirce, J. W. (2007). PsychoPy--Psychophysics software in Python. Journal of Neuroscience Methods, 162(1-2), 8-13. doi: 10.1016/j.jneumeth.2006.11.017

Peirce, J. W. (2008). Generating stimuli for neuroscience ssing PsychoPy. Front Neuroinform, 2, 10. doi: 10.3389/neuro.11.010.2008

Rader, C. M., \& Tellegen, A. (1987). An investigation of synaesthesia. Journal of Personality and Social Psychology, 52(5), 981-987.

Rothen, N., Seth, A. K., Witzel, C., \& Ward, J. (2013). Diagnosing synaesthesia with online colour pickers: Maximising sensitivity and specificity. Journal of Neuroscience Methods, 215(1), 156160. doi: 10.1016/j.jneumeth.2013.02.009

Sandberg, K., Timmermans, B., Overgaard, M., \& Cleeremans, A. (2010). Measuring consciousness: Is one measure better than the other? Conscious Cogn, 19(4), 1069-1078. doi: 10.1016/j.concog.2009.12.013

Simner, J. (2012). Defining synaesthesia. British Journal of Psychology, 103(1), 1-15. doi: $10.1348 / 000712610 X 528305$

Simner, J., \& Bain, A. E. (2013). A longitudinal study of grapheme-color synesthesia in childhood: 6/7 years to 10/11 years. Front Hum Neurosci, 7, 603. doi: 10.3389/fnhum.2013.00603

Simner, J., Harrold, J., Creed, H., Monro, L., \& Foulkes, L. (2009). Early detection of markers for synaesthesia in childhood populations. Brain, 132(Pt 1), 57-64. doi: 10.1093/brain/awn292

Simpson, L., \& McKellar, P. (1955). Types of synaesthesia. Journal of Mental Science, 101, 141-147. 
Sinke, C., Halpern, J. H., Zedler, M., Neufeld, J., Emrich, H. M., \& Passie, T. (2012). Genuine and druginduced synesthesia: A comparison. Conscious Cogn, 21(3), 1419-1434. doi: 10.1016/j.concog.2012.03.009

Studerus, E., Gamma, A., Kometer, M., \& Vollenweider, F. X. (2012). Prediction of psilocybin response in healthy volunteers. PloS one, 7(2), e30800. doi: 10.1371/journal.pone.0030800

Tellegen, A., \& Atkinson, G. (1974). Openness to absorbing and self-altering experiences ("absorption"), a trait related to hypnotic susceptibility. J Abnorm Psychol, 83(3), 268-277.

Terhune, D. B. (2009). The incidence and determinants of visual phenomenology during out-of-body experiences. Cortex, 45(2), 236-242. doi: 10.1016/j.cortex.2007.06.007

Tomson, S. N., Avidan, N., Lee, K., Sarma, A. K., Tushe, R., Milewicz, D. M., . . Eagleman, D. M. (2011). The genetics of colored sequence synesthesia: suggestive evidence of linkage to $16 \mathrm{q}$ and genetic heterogeneity for the condition. Behavioural Brain Research, 223(1), 48-52. doi: 10.1016/j.bbr.2011.03.071

Wapner, S., \& Krus, D. M. (1960). Effects of lysergic acid diethylamide and differences between normals and schizophrenics on the Stroop Color-Word Test. Journal of Neuropsychiatry, 2, 76-81.

Ward, J. (2013). Synesthesia. Annual Review of Psychology, 64, 49-75. doi: 10.1146/annurev-psych$113011-143840$

Witthoft, N., \& Winawer, J. (2013). Learning, memory, and synesthesia. Psychol Sci, 24(3), 258-265. doi: $10.1177 / 0956797612452573$ 